\title{
Microbranching instability in phase-field modelling of dynamic brittle fracture
}

\author{
Jeremy Bleyer* and Jean-François Molinari ${ }^{\dagger}$ \\ Ecole Polytechnique Fédérale de Lausanne \\ Department of Civil Engineering \\ Department of Materials Science \\ 1015 Lausanne, Switzerland
}

\begin{abstract}
The microbranching instability occurring for rapidly propagating cracks in brittle materials has been described in various experiments as an intrinsically three-dimensional phenomenon. Using a variational phase-field model, we show that the microbranching process is, indeed, a threedimensional instability which exhibits a strong dependence on the sample width and can be suppressed for very thin samples. We show that the phase-field internal length scale is the decisive variable governing the branching pattern, which can be either localized in the transverse direction as observed in glass for example, or, on the contrary, almost translational invariant with quasiperiodic structures, as observed in PMMA.
\end{abstract}

Linear elastic fracture mechanics has been shown to describe accurately the dynamics of a single propagating crack at low velocities i.e. below $0.3-0.4 c_{R}$ with $c_{R}$ being the Rayleigh wave speed [1-3]. However, for higher velocities, various experiments demonstrated that cracks stop being simple $[4,5]$ and exhibit dynamic instabilities characterized by crack velocity oscillations and frustrated branching attempts called microbranches [6]. This microbranching instability has been observed in different materials such as PMMA [6], Homalite [7], glass [8] or neo-Hookean brittle gels [9] and present universal features such as the existence of a critical velocity below which branching cannot occur. However, the crack surface patterns can be quite different depending on the material. In glass or gels for instance, microbranches tend to repeat themselves along lines parallel to the crack propagation direction and are highly localized along the width direction of the sample [9]. Conversely in PMMA, at the onset of the microbranching instability, microbranches also localize along the width direction but rapidly merge, forming an almost invariant pattern and showing a periodic alternance of up and down microbranches with a well-defined length scale. This typical length scale does not seem to exist for glass or gels [10]. It is now clear that the microbranching instability is a three-dimensional phenomenon but many aspects such as the origin of the difference in terms of crack surface patterns or the influence of the sample geometry are not fully understood. Our aim is to show through fully three-dimensional simulations that the crack-tip dissipation length scale is the key parameter controlling the different observed branching regimes.

Although microbranching instability has been reproduced numerically using discrete lattice models [11, 12],

\footnotetext{
* Corresponding author: Jeremy Bleyer

jeremy.bleyer@\{epfl.ch,enpc.fr $\}$,

Tel.: +41216932413

† jean-francois.molinari@epfl.ch
}

its occurence seems to be highly dependent on the type of lattice and on the form and intensity of the chosen potential. Similarly, cohesive models are only able to reproduce part of the observed experimental features of dynamic fracture. In particular, cracks do not seem to exhibit a limiting speed below the Rayleigh wave speed despite branching [13]. Besides, the inherent mesh dependency of such an approach makes it ill-suited to an accurate study of the microbranching instability. Other strategies for simulating crack propagation in brittle media consist of non-local continuum models which aim at regularizing the crack topology by a continuous scalar field.

Sharing strong similarities with damage gradient approaches [14], phase-field modelling is a promising method which relies on basic ingredients such as damagedependent elastic moduli, a fracture energy $G_{c}$ and an internal length scale $l_{0}$ (see the Supplemental Materials for more details). The modelling of the I+III mixed-mode instability using such an approach has, for instance, shown very promising results $[15,16]$. Regarding the branching process, a large majority of works concentrated on two-dimensional simulations and, usually, were only able to reproduce macroscopic branching [17-19]. In [20], microbranching has been obtained in 3D computations using a Ginzburg-Landau phase-field model after perturbing the initial crack front and relatively good agreement of the fractographic patterns with experimental observations has been obtained. Although the distinction between macro- and microbranching may be somehow arbitrary, we will consider microbranching as situations where branched crack deviations remain of the order of magnitude of the crack tip dissipation length scale $l_{0}$.

A recent study on the crack branching phenomenon in a two-dimensional pre-strained plate configuration [19] has revealed that single crack propagation is characterized by a progressive widening of the damaged band associated to an increase of the energy release rate. Macroscopic branching has been observed to occur when the energy release rate exceeds a critical value close to $2 G_{c}$, as initially argued by Eshelby [21]. In 


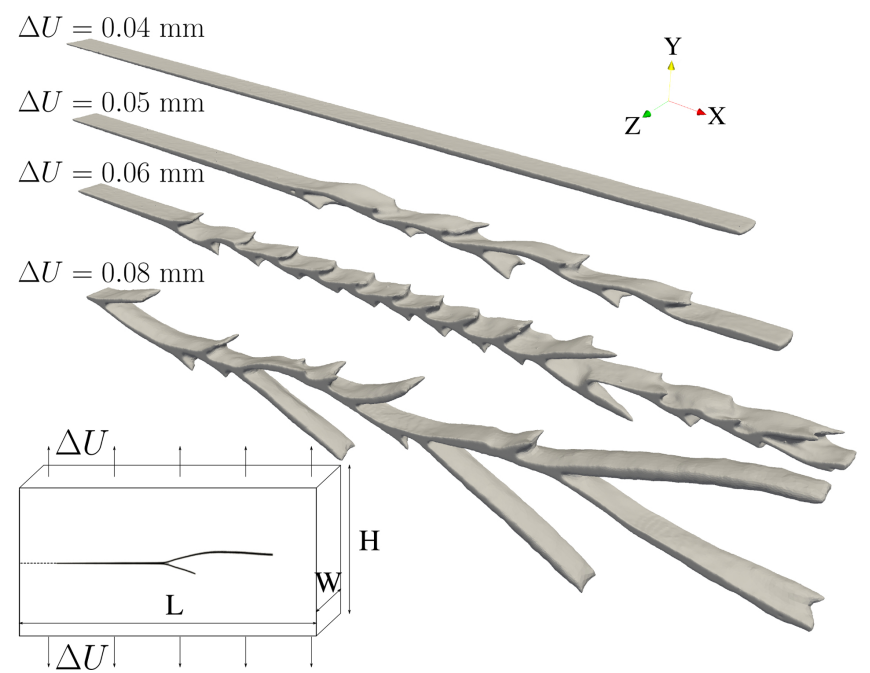

FIG. 1. Crack surface patterns for different loading levels $\Delta U$ for the same plate width $(W=1 \mathrm{~mm})$. The crack propagates as a single straight crack (with a slightly curved crack front) for low loading levels (top), the microbranching instability appears for higher loading with longer branches and shorter distance between successive branches for increasing loading (middle). For even higher loading (bottom), macroscopic branches are formed and are very close to $2 \mathrm{D}$ simulation patterns. Inset: sample dimensions and side view of a typical crack pattern in $2 \mathrm{D}$ simulations.

particular, no microbranching phase has been identified in such simulations for homogeneous media. We aim here at investigating how three-dimensional simulations are able to reproduce this particular instability in the same pre-strained plate configuration.

We considered a finite-element discretization of a rectangular domain of dimensions $L \times H \times W$ in the $(O x y z)$ frame with $L=2 H=32 \mathrm{~mm}$ (see Fig. 1) and the width dimension $W$ ranging from 0.2 to $5 \mathrm{~mm}$, a prenotch of $4 \mathrm{~mm}$ in the $x$-direction is located on the left boundary along the middle plane $y=H / 2$. The internal length scale $l_{0}$ characterizing the phase-field model has also been varied between 0.02 to $0.1 \mathrm{~mm}$, the mesh size being smaller than $l_{0}$ near the main propagation plane to avoid any mesh-dependency. The pre-strain loading consists in fixed vertical displacements of intensity $\pm \Delta U$ on the top and bottom boundaries, both horizontal displacements are free and all other boundaries are stress-free. Unless stated otherwise, the default values are $W=1 \mathrm{~mm}$, $l_{0}=0.04 \mathrm{~mm}$ and $\Delta U=0.06 \mathrm{~mm}$, default material properties are close to PMMA (see [19]). For this specific prestrained plate setting, the initially stored elastic energy per unit length along the $x$-direction is $\Psi=2(\Delta U)^{2} E / H$. We can then define a critical loading $\Delta U_{c}$ below which crack propagation is not possible i.e. when $\Psi=G_{c}$ or equivalently $\Delta U_{c}=\sqrt{G_{c} H / 2 E} \approx 0.028 \mathrm{~mm}$.

The first striking result is that microbranching patterns appear as a transition between single straight crack

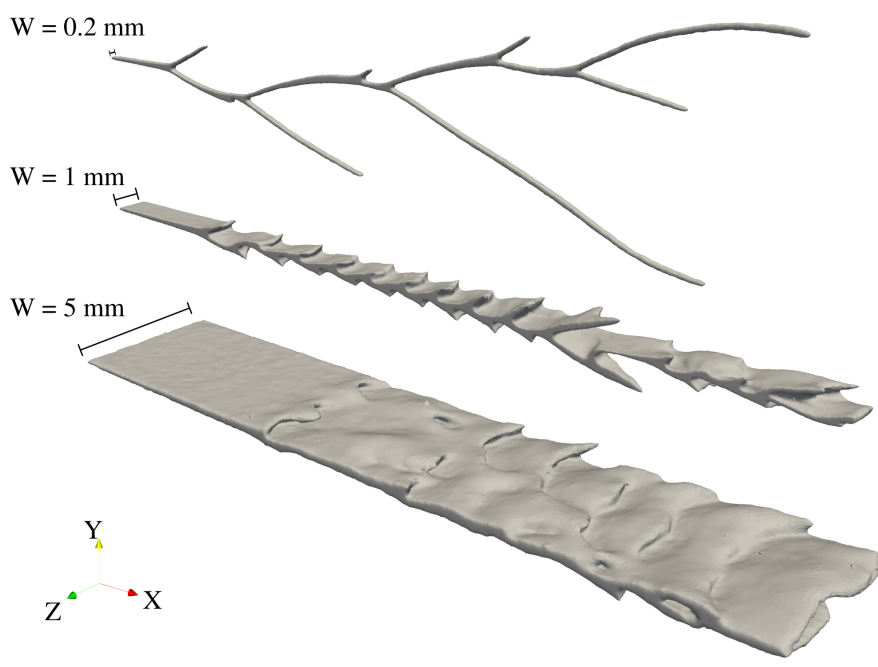

FIG. 2. Crack surface patterns for different plate widths $W$ for the same loading level $(\Delta U=0.06 \mathrm{~mm})$. The microbranching instability is clearly suppressed for the thin plate (top), the macrobranching crack pattern being reminiscent of $2 \mathrm{D}$ simulations. The up-and-down quasi-periodic regime of small microbranching is obtained for the intermediate width (middle) whereas more localization in the $z$-direction is obtained for the larger width (bottom) without exhibiting any well-structured feature. See movies in the Supplemental Materials.

propagation and macroscopic branching patterns (Fig. 1) for three-dimensional simulations with higher stored energy. The patterns obtained for the higher loading $\Delta U=0.08 \mathrm{~mm}=2.9 \Delta U_{c}$ are close to those obtained in $2 \mathrm{D}$ simulations whereas for $\Delta U=0.05$ or $0.06 \mathrm{~mm}$ $\left(1.8 \Delta U_{c}\right.$ or $\left.2.1 \Delta U_{c}\right)$, we observe patterns exhibiting an up-and-down alternance of microbranching attempts which are not seen in $2 \mathrm{D}$ simulations but are very similar to periodic striation patterns appearing in PMMA (see Fig. 4(c) in [6] and [22]). The crack surface for the case $\Delta U=0.06 \mathrm{~mm}$ exhibits more translational invariance along the $z$-direction than the lower loading case $\Delta U=0.05 \mathrm{~mm}$. The increase of symmetry along the $z$-direction with the loading is consistent with experimental observations on PMMA [6] and other simulations using lattice models [12]. In such cases, the crack-tip splitting occurs simultaneously along the whole crack front.

The transition from straight crack propagation, localized events along the $z$-direction and increasing $z$-invariance leading to macroscopic branching seems to be a continuous process with respect to the loading amplitude in which the branch lengths progressively increase. This progressive transition is further confirmed by varying the specimen width $W$ for the same loading (Fig. 2). The microbranching instability can be suppressed by decreasing the plate width up to $W=0.2 \mathrm{~mm}$ for this loading. The suppression of the microbranching instability for very thin plates has also 
been reported experimentally for brittle gels [23]. In these experiments, a crack-tip oscillatory instability has been observed for velocities higher than $0.9 c_{s}\left(c_{s}\right.$ being the shear wave speed), which we do not obtain here and observe macrobranches instead. The question remains to know if this difference can be attributed to nonlinear geometric effects due to the large prestrain level for such gels. Conversely, when increasing the plate width up to $5 \mathrm{~mm}$, the crack surface exhibits much more $z$-localized events without any well defined quasi-periodic patterns as those for $W=1 \mathrm{~mm}$. By increasing the loading for $W=5 \mathrm{~mm}$, we confirm that the crack surface becomes increasingly $z$-invariant with the same quasi-periodic microbranching regime. Contrary to [20], we did not observe a strong difference in terms of critical speed associated with branching compared to the 2D simulations of [19]. Interestingly, the crack branching speeds have always been observed to be higher than the theoretical minimal speed of $0.52 c_{R}$ necessary for the propagation of branched cracks found in [24].

By looking closely at the initiation of the instability for the largest width $W=5 \mathrm{~mm}$, the initial propagation phase corresponds to a single flat crack surface with a slightly curved crack front due to 3D effects. The fully damaged zone increases with crack advance, as in 2D simulations, which can be associated to an increasing local damage dissipation rate $\Gamma$, identified with an apparent fracture energy (see [19]). We computed this quantity for different two-dimensional slices along the $z$-direction and at different propagation times. We obtained the local variation of $\Gamma$ by mapping these values to the current position of the main crack front along the middle plane $Y=H / 2$. The evolution of $\Gamma(x, z)$ along with the crack front position at different time intervals are represented in Fig. 3-top. The initial flat propagation phase corresponds to a uniform increase of $\Gamma$ with respect to $z$ until approximately $6 \mathrm{~mm}$ from the prenotch. Between 6 and $8 \mathrm{~mm}$, slight variations of $\Gamma$ along $z$ appear and the first microbranching events take place at locations where $\Gamma(x, z)$ exceeds $2 G_{c}$, splitting the front into two different parts. Between the branching events, $\Gamma$ is smaller and the single crack front continues its propagation. The coexistence of these two different states along the $z$-direction leads to a strong curvature of the crack front and generates complex dynamics with important variations of crack velocity and energy release rate (Fig. 3-bottom). It is worth noting that $\Gamma$ shows a non-monotonic behavior with respect to the crack front velocity prior to branching. This can be explained by the fact that the increasing damage dissipation (although the crack front has not branched yet) is compensated by a slowdown of the crack front. This slight slowdown of the crack front prior to branching has also been observed in [20]. As described in [25], the release of the crack front from the branch arrest leads to an increase of crack velocity and apparent fracture energy ahead of the first event and eventually forms a new branching event along
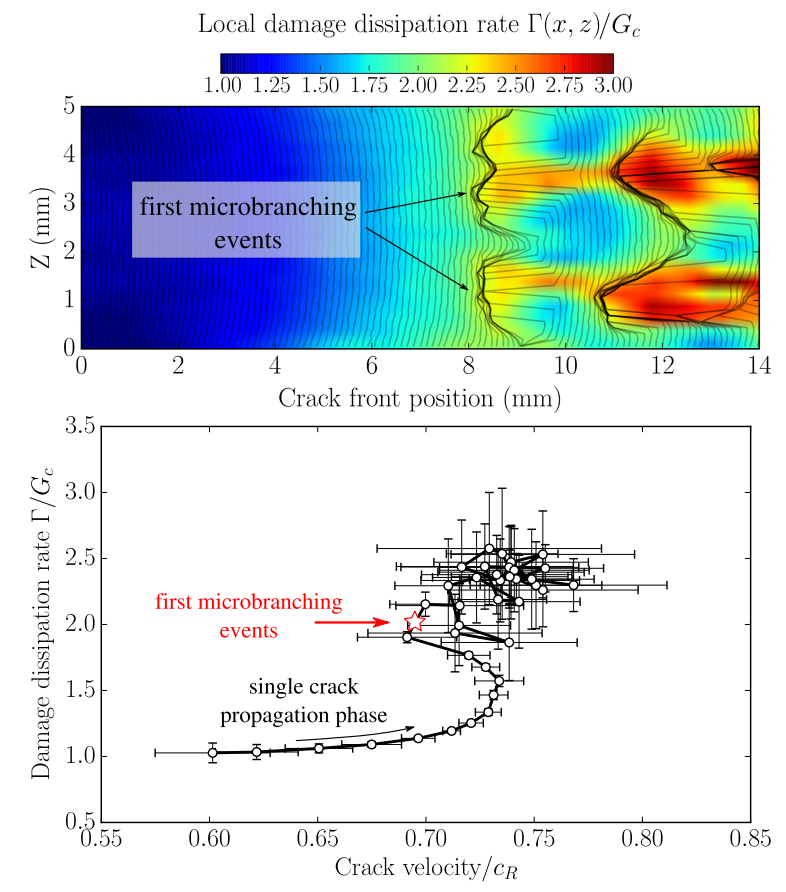

FIG. 3. Top: Evolution of local damage dissipation rate $\Gamma(x, z)$ with crack front advance for $W=5 \mathrm{~mm}(\Delta U=0.06$ $\mathrm{mm})$, the thin lines correspond to the crack front position at successive identical time intervals. The first microbranching events occur at localized regions along the $z$-direction where $\Gamma$ exceeds $2 G_{c}$ and result in large local deceleration and a distorted crack front along the main plane. The second branching events occur along the same lines as the first ones associated to an increase of damage dissipation along these preferential directions. Bottom: Evolution of average crack velocity $v$ and damage dissipation rate $\Gamma$ along the crack front during propagation. For the single crack propagation phase, the standard deviation along the crack front is small whereas large variations occur during the microbranching phase due to non-uniformity of $v$ and $\Gamma$ along the front.

the same direction.

The robustness of the previous results has been assessed by introducing some randomness on the local Young's modulus or on the local fracture energy $G_{c}$ in the sample (the standard deviation was about $10 \%$ ). The quasi-periodic regime and, in particular, the microbranches length $L_{\text {branch }}$ and their typical spacing along the $x$-direction $\Delta x$ did not change. However, the randomness triggers the first branching event at different time and location. It also modifies the dynamics for the $z$-localized patterns (for $W=5 \mathrm{~mm}$ in particular), resulting in a different fracture surface. These results confirm that the quasi-periodic regime and, in particular, $L_{\mathrm{branch}}$ and $\Delta x$, are weakly coupled to $3 \mathrm{D}$ dynamics in the $z$ direction, which differs when adding randomness. In [10], these quantities have been shown to scale with a materialdependent length $l_{1}=G_{c}(v) / E$ or $l_{2}=G_{c}(v) E / \sigma_{c}^{2}$ where $E$ is Young's modulus and $\sigma_{c}$ a critical stress. In our phase-field framework, the material fracture energy is 
rate-independent $G_{c}(v)=G_{c}$ and $l_{0}$ can be related to the following elastic limit stress $\sigma_{c}=\sqrt{3 G_{c} E /\left(8 l_{0}\right)}[14]$, so that $l_{2}=8 l_{0} / 3$. We then tested the previous scaling laws by first varying the ratio $l_{1}=G_{c} / E$ while keeping $l_{0}$ constant and secondly by varying $l_{0}$ while keeping $l_{1}$ constant. In the first case, we changed either $G_{c}$ (resp. $E$ ) while keeping $l_{0}$ and $E$ (resp. $G_{c}$ ) fixed. In order to compare similar situations with the same crack driving force, we also changed the loading level $\Delta U$ to keep the same ratio between the initial stored energy per unit length $\Psi$ and $G_{c}$. The results in terms of crack patterns are exactly the same suggesting that $l_{1}$ is not the governing length scale of the microbranching instability in the phase-field model. On the contrary, when varying $l_{0}$, we observed a clear dependence of all the crack patterns for various loadings and plate widths: in particular, $L_{\text {branch }}$ and $\Delta x$ in the quasi-periodic regime increase with $l_{0}$ (Fig. 4). By recording these quantities for many patterns exhibiting this quasi-periodic regime we show, first, that $L_{\text {branch }}$ and $\Delta x$ are positively correlated, suggesting that the nucleation of the next branching event is directly related to the death of the previous one as proposed in [25]. Moreover, the plate width $W$ seems to have no influence on selecting the microbranch length scale contrary to $l_{0}$. More precisely, we find that the mean values of branch length and periodicity scale are given by $\left\langle L_{\text {branch }}\right\rangle=(20.5 \pm 2.5) l_{0}$ and $\langle\Delta x\rangle=(12.8 \pm 1.7) l_{0}$ for the data of Fig. 4 .

Increasing $l_{0}$ for fixed geometry and loading progressively leads to macroscopic branching as the branches get longer and farther apart. On the contrary, reducing $l_{0}$ leads to smaller and closer microbranches but also less $z$-invariance, as when increasing the plate width. Quite interestingly, the total dissipated energy for the three patterns illustrated in Fig. 4 is the same within $2 \%$. Although a precise quantitative investigation is difficult, our simulations indicate that the typical width of a branching event $\Delta z$ is also positively correlated to $l_{0}$ and therefore explains that translational $z$-invariance appears when $l_{0} / W \propto \Delta z / W$ becomes close to unity, thus separating the two microbranching regimes. revHowever, $\Delta z$ is much larger than $l_{0}$ by at least one order of magnitude so that the transition occurs at a macroscopic width compared to $l_{0}$. Unfortunately, larger simulations are out-of-reach to further investigate the influcence of $W$ with respect to the extent of localized events in the $z$-direction.

These different results therefore suggest that the microbranching instability patterns are highly dependent on an intrinsic material length scale characterizing the dissipation processes at the crack tip. In the phasefield framework, this length scale is given by $l_{0}$ and can be interpreted as a cohesive zone length over which the LEFM singularity is regularized. Consequently, as a crack front accelerates, the apparent fracture energy increases through a thickening of the fully damaged band which we associate with a velocity-toughening mechanism. When reaching locally a critical value of $2 G_{c}$, crack

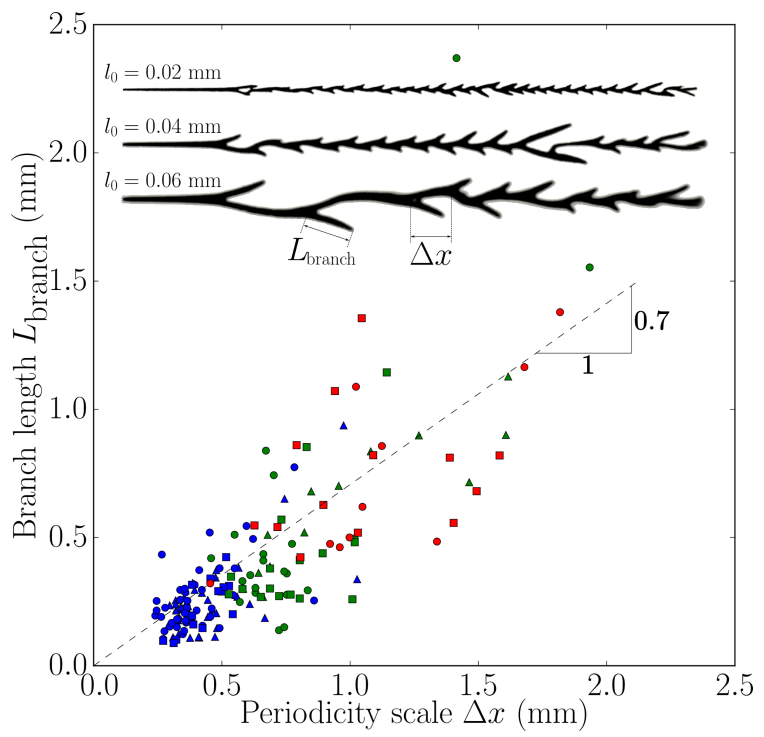

FIG. 4. Correlation between microbranch spacing $\Delta x$ and microbranch length $L_{\text {branch }}$ in quasi-periodic regime for different $W(\triangle: 0.5 \mathrm{~mm}, \circ: 1 \mathrm{~mm}, \square: 2 \mathrm{~mm})$ and different $l_{0}$ (blue : $0.02 \mathrm{~mm}$, green : $0.04 \mathrm{~mm}$, red : $0.06 \mathrm{~mm}$ ). Inset: side view of $z$-invariant patterns for $W=1 \mathrm{~mm}$ and different $l_{0}$.

tip splitting occurs over a width $\Delta z$. If $\Delta z / W \leq 1$, splitted and single crack tips can coexist simultaneously along the crack front. This results in complex dynamics in the $(x, z)$-plane due to crack front curving and release of energy when the microbranch dies. This situation typically occurs in glass for which the critical length scale is very small compared to the typical sample size. Reproducing such localized chains of microbranching is unfortunately too computationally expensive as it would require an extremely fine mesh to resolve the small length scale $l_{0}$ but our results in the extreme cases with the smallest $l_{0} / W$ confirm this scenario. Conversely, if $\Delta z / W \geq 1$, crack tip splitting occurs along the whole front and the crack tip dynamics enter a new stable regime of alternating up-and-down microbranches with well defined geometric characteristics related to $l_{0}$. This situation typically occurs in PMMA for which the characteristic length scale is much higher than for glass. When $\Delta z / W \gg 1$, this regime translates into macroscopic branching as branched crack tips are now sufficiently well separated and are less influenced by each other.

Obviously, the previous analysis also depends on the value of the loading or crack velocity although the main ideas still hold. A different crack velocity changes the fracture energy in PMMA for instance and, therefore, influences the length scale. Here, we used a rateindependent phase-field model which does not account for a material dependence of $G_{c}$ on the velocity. Most certainly, the very simple damage model used in the phase-field approach misses some complex mechanisms taking place inside the crack tip process zone, explaining 
why the obtained crack front velocities are relatively high (around $0.7 c_{R}$ ) compared to critical velocities measured experimentally. However, the results of Fig. 3 show that the onset of branching is well discribed by a local energetic criterion on $\Gamma$. Hence, for high loading or crack velocity, there is a higher probability that random perturbations will surpass this critical value, yielding a larger width $\Delta z$ of the microbranching event, until eventually reaching a full localization across the sample width. Similarly, at higher loading level, branched crack tips release more energy and may survive longer than for configurations with less energy, resulting in a transition to macroscopic branching.

In conclusion, our simulations reveal a clear 2D3D-2D transition from a straight crack propagation to $z$-localized microbranching, further evolving into a $z$-invariant quasi-periodic regime and eventually into macrobranching, in good agreement with the well-known mirror-mist-hackle transition observed on post-mortem fracture surfaces. We have shown how the material length scale $l_{0}$ governs the different branching regimes with respect to the specimen width and the loading level. In particular, the microbranching regime cannot be obtained for very thin samples or $2 \mathrm{D}$ simulations due to its intrinsic three-dimensional nature. The onset of this dynamic instability is noise-triggered and occurs when the local energy release rate exceeds a critical value of $2 G_{c}$, already associated to the onset of branching in $2 \mathrm{D}$ simulations. Depending on the size of the typical branching width with respect to the sample width, branching patterns are either translationally invariant and weakly coupled to 3D dynamics as observed in PMMA or localized along the $z$-direction and highly dependent on complex dynamics, which is close to what is observed in glass and brittle gels. The present work illustrates that phase-field models are a relevant approach for modelling complex crack propagation dynamics, relying on simple well-defined energetic principles. More quantitative results (especially concerning crack velocities) can be expected by modelling rate-dependency in PMMA. Finally, better insight on the branch width selection could be gained from theoretical perturbation analysis of crack-tip splitting processes.

Details regarding the phase-field model used for the simulations are given in the supplemental material attached with this paper.
[1] L. B. Freund, Dynamic fracture mechanics (Cambridge university press, 1998).

[2] E. Sharon and J. Fineberg, Nature 397, 333 (1999).

[3] E. Bouchbinder, T. Goldman, and J. Fineberg, Reports on Progress in Physics 77, 046501 (2014).

[4] K. Ravi-Chandar, Dynamic fracture (Elsevier, 2004).

[5] J. Scheibert, C. Guerra, F. Célarié, D. Dalmas, and D. Bonamy, Physical Review Letters 104, 045501 (2010).

[6] E. Sharon and J. Fineberg, Physical Review B 54, 7128 (1996).

[7] K. Ravi-Chandar and W. Knauss, International Journal of Fracture 26, 141 (1984).

[8] E. Sharon and J. Fineberg, Philosophical Magazine B 78, 243 (1998).

[9] A. Livne, G. Cohen, and J. Fineberg, Physical Review Letters 94, 224301 (2005).

[10] E. Bouchbinder and I. Procaccia, Physical Review E 72, 055103 (2005).

[11] S. I. Heizler, D. A. Kessler, and Y. S. Elbaz, Physical Review E 88, 022401 (2013).

[12] S. I. Heizler and D. A. Kessler, Physical Review E 92, 012403 (2015).

[13] F. Zhou, J.-F. Molinari, and T. Shioya, Engineering
Fracture Mechanics 72, 1383 (2005).

[14] K. Pham, H. Amor, J.-J. Marigo, and C. Maurini, International Journal of Damage Mechanics 20, 618 (2011).

[15] A. J. Pons and A. Karma, Nature 464, 85 (2010).

[16] H. Henry, EPL (Europhysics Letters) 114, 66001 (2016).

[17] A. Karma and A. E. Lobkovsky, Physical Review Letters 92, 245510 (2004).

[18] H. Henry, EPL (Europhysics Letters) 83, 16004 (2008).

[19] J. Bleyer, C. Roux-Langlois, and J.-F. Molinari, International Journal of Fracture in press, 1 (2016).

[20] H. Henry and M. Adda-Bedia, Physical Review E 88, 060401 (2013).

[21] J. Eshelby, in Fundamental Contributions to the Continuum Theory of Evolving Phase Interfaces in Solids (Springer, 1999) pp. 82-119.

[22] E. Bouchbinder, J. Mathiesen, and I. Procaccia, Physical Review E 71, 056118 (2005).

[23] A. Livne, O. Ben-David, and J. Fineberg, Physical Review Letters 98, 124301 (2007).

[24] M. Adda-Bedia, Journal of the Mechanics and Physics of Solids 53, 227 (2005).

[25] I. Kolvin, G. Cohen, and J. Fineberg, Physical Review Letters 114, 175501 (2015). 